\title{
Properties of Filamentary Sublimation Residues from Dispersions of Clay in Ice
}

\author{
R. STEPHEN SAUNDERS, ${ }^{*}$ FRASER P. FANALE, $\uparrow$ TIMOTHY J. PARKER,* \\ JAMES B. STEPHENS,${ }^{*}$ AND STEPHEN SUTTON $\ddagger$
}

\author{
*Jet Propulsion Laboratory, California Institute of Technology, MS 183-501, 4800 Oak Grove Drive. \\ Pasadena, California 91109; $¥$ University of Hawaii, 2525 Correa Road, Honolulu, Hawaii 96822; and \\ †University of Michigan, 600 East Kingsley, Apartment 3, Ann Arbor, Michigan 48104
}

Received July 17, 1985; revised December 2, 1985

\begin{abstract}
During vacuum sublimation experiments on simulated Martian polar deposits and cometary dirty ices, a fuffy filamentary sublimate residue material with unique physical properties was produced. The silica-to-silica bonds that we believe join the particles together are the result of conditions that may exist in some Martian polar deposits and on some cometary surfaces. Submicron particles of montmorillonite clay thinly dispersed ( $1: 1000$ clay/water) and not contacting one another in water ice can form very-low-density structures (density as low as $10^{-3} \mathrm{~g} \mathrm{~cm}{ }^{3}$ ) during sublimation of the ice. The lightweight constructs, when viewed in scanning electron microscopy micrographs, are composed of long network chains of the clay particles. The material is sufficiently electrically conductive to drain away the scanning electron microscopy charge. It is also resistant (no change in electronic properties are apparent) to scanning electron microscopy electron-beam heating for hours in vacuo. Infrared spectra and $\mathrm{X}$-ray diffraction patterns of the sublimate residues show little difference from spectra and patterns of the original minerals. Heating in an oven, in air, to $370^{\circ} \mathrm{C}$ produces little change in the structure of the sublimate residual material. The particle bonding forces are strong and produce a resilient, elastic lightweight material. The material is porous and will allow vapors to diffuse through it, and its thermal conductivity is very low. These properties produce a high-performance vacuum insulation. This material may have applications for insulating ice bodies (solid cryogens) in space. The incoming heat is partially carried away by the out-flowing water vapor. 1986 Academic Press. Inc.
\end{abstract}

\section{INTRODUCTION}

Geologic studies of the surfaces of Solar System objects rely on various types of remote sensing, laboratory simulations, and theoretical modeling. This paper describes some of the laboratory experiments performed in support of our studies of surfaces of materials in which a major component is water ice mixed with mineral particles. Our objective is to characterize such icy materials and evaluate them as candidate substrates of various natural surfaces.

The particular experiments reported here were designed to examine sublimate residues of dispersions of clay particles in ice. The properties of sublimate residues are of interest in understanding the geologic processes that may be active within the polar deposits of Mars, on the surfaces of the icy satellites, and on comet nuclei.

In the Martian and comet environments in particular, the low-pressure and lowtemperature sublimation of water ice containing various amounts of dispersed particulate matter is thought to be an important process (Cutts et al., 1979; Brownlee, 1978). In these materials it is believed that dust particles acted as nucleation sites for the accumulation of ices during the initial formation process. The result of this nucleation would be a complex ice in which the dispersed dust particles are not in contact (Whipple, 1978). Our experiments show that under the environmental conditions that exist on Mars and comets, the water ice would sublime away, and that an open filamentary aggregate of fine particles may 
mantle the ice surface, or that these particles may be released as dusts. The properties of such sublimate residue mantlestheir densities, the size and shape of any aggregates, and the degree and type of cohesion between particles and particle aggregates-are discussed in this paper. These particle aggregates are of importance in the thermal and sedimentation modeling of Martian and cometary ice surfaces (Scheidegger, 1974; Fanale and Salvail, 1984) and may provide clues to the geologic processes that produce certain features such as cometary mantles or Martian circumpolar dunes. The properties of the dust plumes that may be formed will be discussed at a later time.

To understand these properties, a series of low-pressure and low-temperature experiments was conducted during which the water ice was sublimed from dilutions, of various ratios, of frozen water-clay dispersions.

\section{EXPERIMENTS AND OBSERVATIONS}

The experimental dispersions were prepared by mixing distilled water with airclassified montmorillonite clay (size $<0.2$ $\mu \mathrm{m}$ ) in weight ratios of $100: 1$ and $1000: 1$. These mixtures were sprayed into an openmouth dewar of liquid nitrogen. The individual droplets froze very quickly upon contact with the liquid nitrogen (Fig. 1). The rate of freezing is a strong function of the droplets' size. By minimizing the size of the spray nozzle and maximizing the atomizing pressure, the droplets can be reduced to micron size. Coarser sprays are produced from larger nozzles at low pressures. The slurry of liquid nitrogen and ice droplets in the dewar was then placed in a vacuum chamber capable of reaching pressures below $15 \mathrm{mTorr}$ ( $20 \mu$ bar).

The sublimation process takes several days to complete and requires more time with larger quantities of slurry, particularly if the resultant filamentary residue is very low in density (i.e., a good insulator). The final temperature of the ice at depletion was

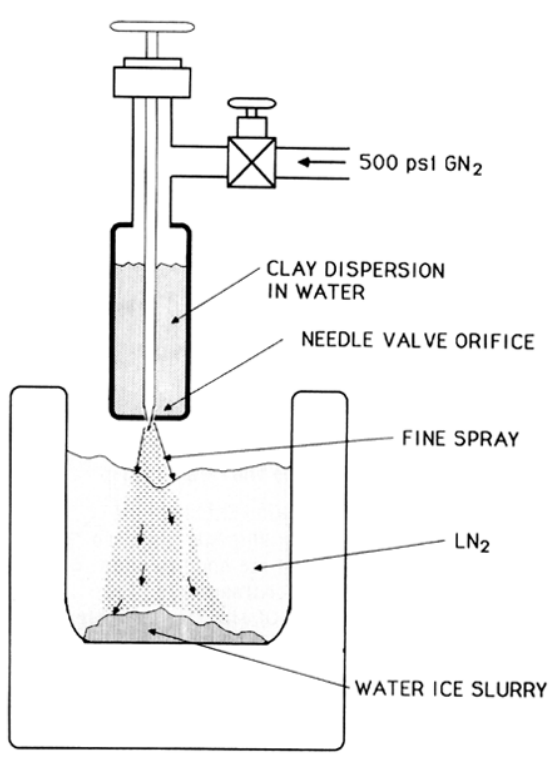

FIG. 1. High-speed freezing of fine-spray droplets to produce noncontacting dispersion of clay particles in ice.

lowest (about $-50^{\circ} \mathrm{C}$ ) for the experimental runs that produced the lowest density filamentary material (Fig. 2) but, because the thermocouple lead wire was a relatively large source of heat, the ice would always sublime away from it so that the measured temperatures were only relative. A lowdensity construct of sublimate residue remained on the bottom of the dewar after complete sublimation of the water ice.

Experimental results with two different dilutions of montmorillonite and one of $\mathrm{Ca}$ bosil in water are given in Table I. This table represents results from over $\mathbf{3 0}$ different experimental runs and demonstrates the variability of the properties of the individual sublimate residual materials. All of the sublimate residues formed aggregates capable of supporting their own weight. They are also cohesive; two fragments will adhere to one another when brought into light contact.

Optical and scanning electron microscopy (SEM) reveal that the sublimate residue 100:1 water-clay mixtures consist of botryoidal (grape-cluster-like) aggregates of 0.1 - to $200-\mu \mathrm{m}$-diameter spheres with a 


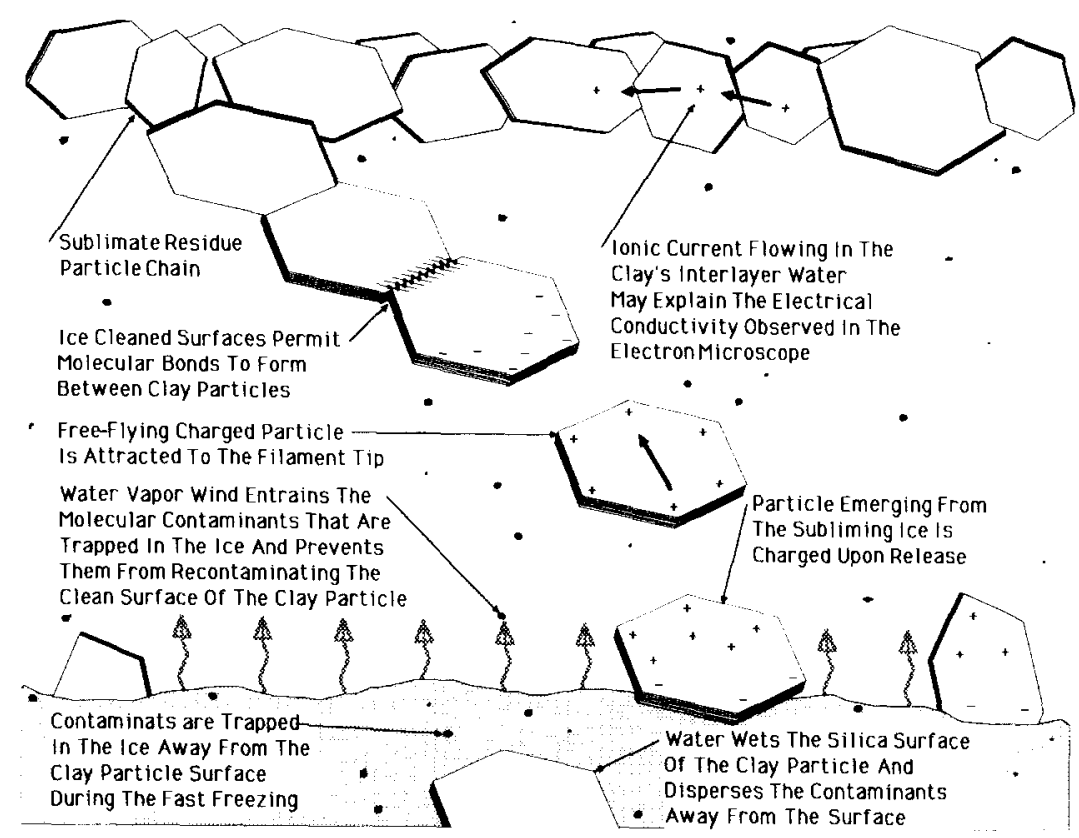

Fig. 2. "Ice-cleaned" clay particle just after release from subliming ice surface and before contact with the filamentary particle chain. Water vapor wind entrains the contaminants and carries them away from the clay particle during the sublimation process.

density of $3 \times 10^{-3} \mathrm{~g} \mathrm{~cm}^{-3}$ (Fig. 3); presumably, this is the size and shape of the coarse water-clay droplets from the sprayer. The presence of expansion cracks and vent holes on the surfaces of most spheres indicates that the outer layer of a droplet freezes very quickly upon contact with the liquid nitrogen and is then ruptured by the expansion of the sphere or by the release of trapped dissolved gases. Radial tube-like or open trilete, petal-shaped structures are common in these relict spheres.

The filamentary sublimate residue formed by freeze-drying the $1000: 1$ dispersion does not retain the original spherical shape of the fine spray droplets; instead it consists of an open network of clay particles (Fig. 4). Reconfiguration of the clay particles into filamentary particle chains as this material is sublimed is indicated both

TABLE I

Summary of Physical Properties of Sublimate Residues

\begin{tabular}{|c|c|c|c|c|c|}
\hline Particulate material & Dispersion dilution & $\begin{array}{l}\text { Density } \\
\left(\mathrm{g} / \mathrm{cm}^{3}\right)\end{array}$ & Cohesive nature & Mechanical strength & SEM appearance \\
\hline $\begin{array}{l}\text { Montmorillonite } \\
\text { clay }\end{array}$ & $1000: 1$ fine spray & $\begin{array}{l}.0009 \text { "Filamentary } \\
\text { residue" (Fig. 4) }\end{array}$ & $\begin{array}{l}\text { Rebond nearly } \\
\text { as good as } \\
\text { original }\end{array}$ & Tough and elastic & $\begin{array}{l}\text { Filamentary } \\
\text { particulate } \\
\text { chains of clay }\end{array}$ \\
\hline $\begin{array}{l}\text { Montmorillonite } \\
\text { clay }\end{array}$ & $100: 1$ coarse spray & $\begin{array}{l}.003 \text { "Botryoidal } \\
\text { residue" (Fig. 3) }\end{array}$ & $\begin{array}{l}\text { Less cohesive } \\
\text { than } 1000: 1\end{array}$ & $\begin{array}{l}\text { Less tough and } \\
\text { elastic than } \\
1000: 1\end{array}$ & $\begin{array}{l}\text { (Relict shape of } \\
100-\mu \mathrm{m} \text { coarse } \\
\text { water droplets) }\end{array}$ \\
\hline $\begin{array}{l}\text { Cabosil (glass } \\
\text { smoke) }\end{array}$ & $1000: 1$ fine spray & .007 & $\begin{array}{l}\text { Less than } \\
\text { above }\end{array}$ & $\begin{array}{l}\text { Much more friable } \\
\text { than others }\end{array}$ & $\begin{array}{l}\text { Filamentary } \\
\text { chains of glass } \\
\text { smoke } \\
\text { particles }\end{array}$ \\
\hline
\end{tabular}



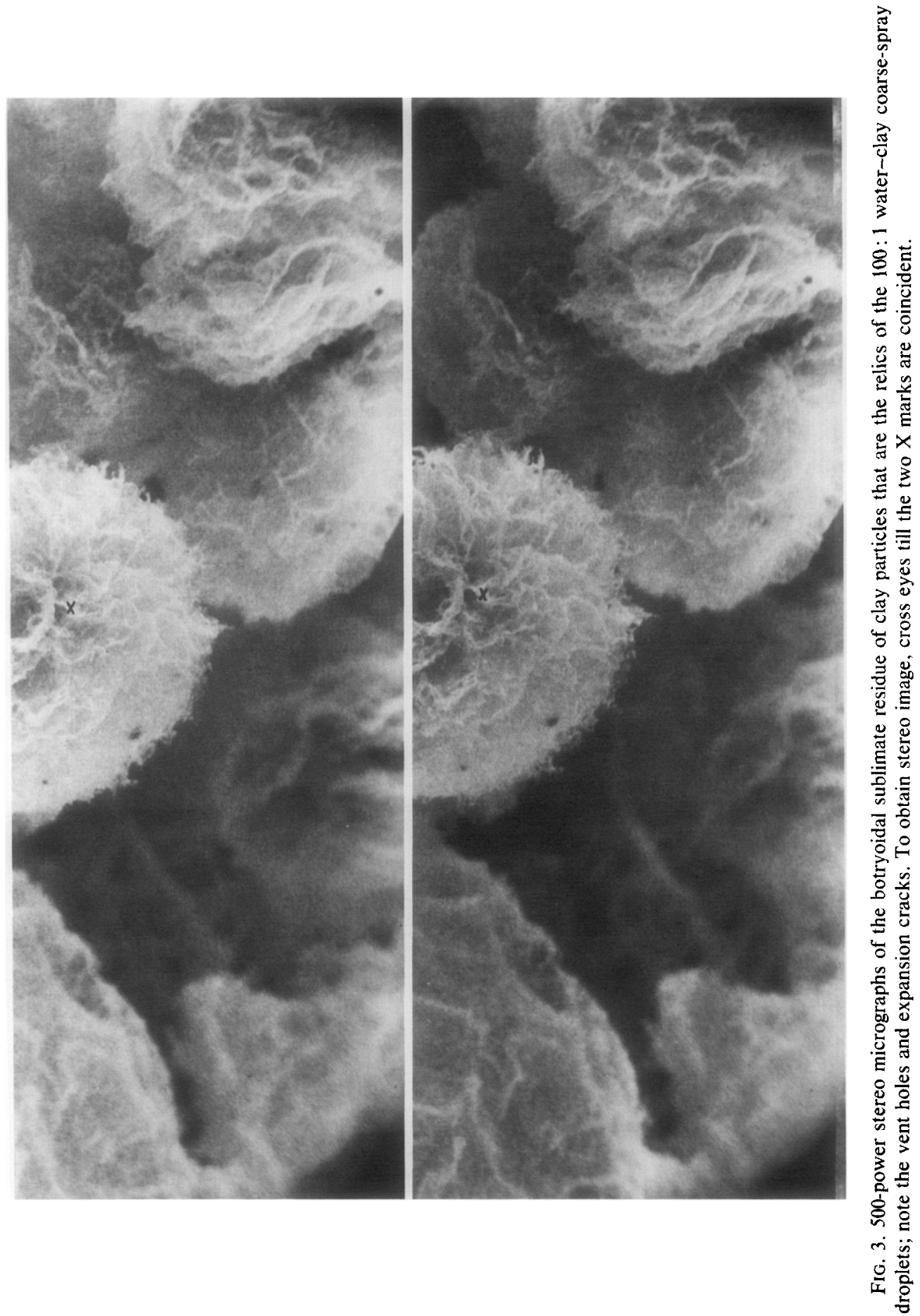


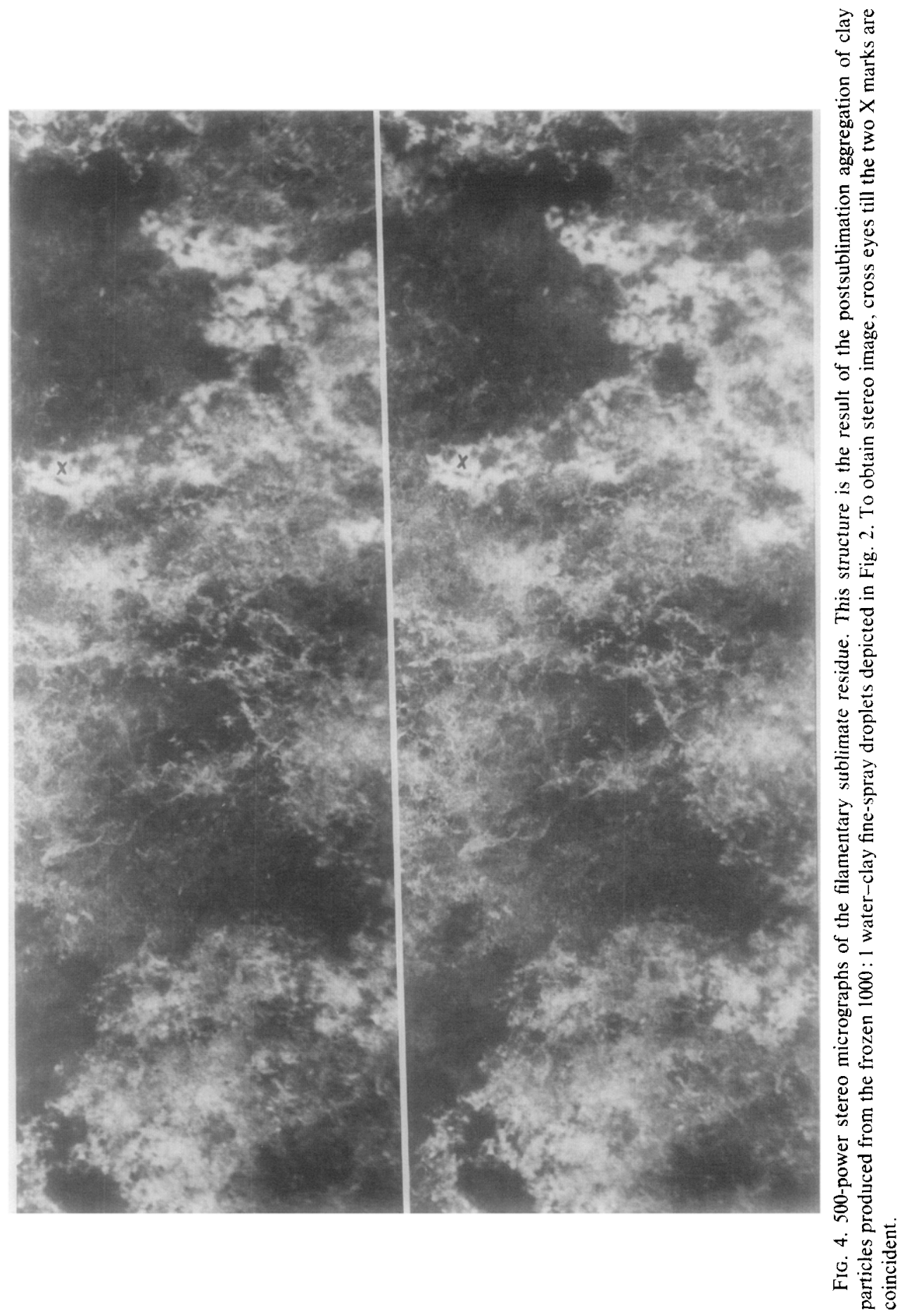


by the change in morphology (nonretention of the spherical shapes) and substantial reduction in bulk density over that which could be expected if the clay particles had maintained their original positions in the ice. If this were the case, the density would be $3 \times 10^{-3} \mathrm{~g} \mathrm{~cm}^{-3}$ as in the botryoidal case. If the particles had not reorganized themselves into interconnected filamentary chains upon release from the ice, they would have settled to the surface of the sublimation container. This is in fact what happens upon sublimation of nonsiliceous materials that do not form filamentary chains (see below). The filamentary residue material from the 1000:1 montmorillonite dispersion behaved elastically even at 15 mTorr (determined by vibrating the vacuum chamber and observing the oscillations of the filamentary sublimate residue overhangs). The formation of blisters in the filamentary sublimate residue layer during the sublimation process is further evidence that the bonds between clay particles are tough and flexible. It also indicates that there is little or no adhesion between the ice and the sublimate residues forming above it. A more realistic "dirty" ice, however, might be expected to have rod-like particles and aggregates that would maintain a tensile attachment between the subliming ice and the sublimate residue (Brownlee, 1978).

Compressive stress-strain measurements of the filamentary sublimate residue give an indication of the elastic-plastic behavior of the aggregate material. A repeating of the stress excursion in the elasticplastic portion of the stress-strain curve proves the material's inherent elastic properties even though some hysteresis was evident. The compressive elastic limit for two of the filamentary sublimate residue samples was in the range of $10^{3} \mathrm{dyn}^{-2}$ at a strain of about $50 \%$.

The densities of several samples of the 1000: 1 filamentary sublimate residue were determined by weighing several lumps larger than $1 \mathrm{~cm}^{3}$ and estimating their vol- umes. The average density of the $1000: 1$ sublimate residue averaged $0.002 \mathrm{~g} \mathrm{~cm}^{-3}$. At the low-density extreme, a specific gravity of $0.0009 \mathrm{~g} \mathrm{~cm}^{-3}$ was obtained from one lump that was over $10 \mathrm{~cm}^{3}$ in volume. The typical range for the measured samples, however, was from 0.001 to $0.005 \mathrm{~g} \mathrm{~cm}^{-3}$ (the higher density may be due to absorbed water). The 1000:1 Cabosil filamentary sublimate residue was $0.007 \mathrm{~g} \mathrm{~cm}^{-3}$.

The formation of a less cohesive and more friable sublimate reside using submicron spherical particles of amorphous silica (Cabosil) demonstrates that other materials can form similar filamentary particle chain structures with somewhat different characteristics (see Table I). The relative weakness is possibly due to the nature of the bonds at the spherical contact points. The area of contact between the spherical particles of the Cabosil is smaller than the area between the crystalline facets of the clay particles.

Clay particles are composed of lamellar structures that can hold water between the layers. To investigate what effect the presence or absence of interlayer water might have on the properties of the clay aggregates, the following tests were performed on the 1000:1 filamentary sublimate residue. The filamentary aggregate structure underwent no visible change after more than $16 \mathrm{hr}$ in the $5 \times 10^{-5}$ Torr vacuum of the SEM. A 30-kV 800-picoamp SEM electron beam rastered over a small area (approximately $1 \mu \mathrm{m}^{2}$ ) of the material for $3 \mathrm{hr}$ caused no observable change in conductivity, build-up of charge, or structural damage. The aggregate must therefore be electrically conductive to some extent. In addition to the SEM experiments, it was shown that heating at $181^{\circ} \mathrm{C}$ at 10 Torr (13 mbar) for $80 \mathrm{hr}$ in a vacuum oven and then in air to $370^{\circ} \mathrm{C}$ had no noticeable effect on the material's properties. Since temperatures from 120 to $200^{\circ} \mathrm{C}$ are sufficient to drive off the interlayer water from montmorillonite (Weaver and Pollard, 1973), persistence of cohesion in the sample is not 
dependent on the continual presence of interlayer or surface water.

Several control experiments were conducted in order to verify that formation of this filamentary sublimate residue is unique to the above conditions. In the first control experiment, a "dry" mixture of montmorillonite and water ice (in which no liquid water was ever in contact with the clay) was mixed together in liquid nitrogen. This yielded a material that, upon freeze-drying, resembled the dry, unprocessed montmorillonite clay. This suggests that the clay particles form the more elaborate and larger aggregates only when dispersed within the subliming water ice. In other words, intimate contact between clay and water seems to be required at some point in order for strong filamentary or botryoidal aggregates to form-at least in the time provided for in laboratory tests.

As additional control experiments, $1000: 1$ dispersions of other fine particulate materials (the minerals calcite and forsterite, and industrial iron oxide, alumina, graphite, and carbon black) that have intrinsically lower solubilities in water than montmorillonite were each sprayed into liquid nitrogen and then freeze-dried in the same manner as the montmorillonite and Cabosil. Each of these materials formed a thin nonfilamentary, nonfluffy residue on the bottom of the sublimation vessel.

Four additional control experiments were made. Slurries of $75-\mu \mathrm{m}$-diameter $\mathrm{SiO}_{2}$ glass beads were processed in a manner similar to the clay solutions, but the resulting material was little different from the unprocessed beads. Dry montmorillonite was mixed with liquid nitrogen and placed in the vacuum chamber; the resulting material exhibited no change in cohesion and an only slightly lower density than unprocessed montmorillonite. Microscopically, it was very similar to unprocessed montmorillonite. Next, a 950:1 mixture of deionized water and montmorillonite was dried for $21 \mathrm{hr}$ in an oven at atmospheric pressure and temperatures between 83 and $88^{\circ} \mathrm{C}$. The dried clay formed a thin film over the surface of the container and was hard and brittle. And finally, a 100:1 solution of water and montmorillonite was frozen and then freeze-dried. The structure of the sublimate residue was a relic of the growth interference faces of the ice crystals. The slowly freezing ice "zone refined" the clay particles into petal-like structures that represented the boundaries between the individual ice crystals.

\section{DISCUSSION}

In the experiments that we have performed, we have discovered a phenomenon that may be of interest in the study of processes that occur on the surfaces of ice bodies in the outer Solar System. Our experiments indicate that geometrically different cohesive sublimate residues can be formed from the same ingredients by varying the process. For silicate materials, solubility product may play a role in determining the strength of the bond between particles that form the sublimate residue. However, the behavior of calcite demonstrates that solubility is not the only factor. Calcite particles were much larger, which may explain why they did not form the filamentary sublimate residue structure.

The nature of these particle-to-particle bonds are of primary interest. The role of solubility and other physical and chemical considerations require additional study. This initial phase of the work has only demonstrated a process that could occur with materials and conditions that may exist on surfaces of Solar System objects.

A filamentary, cohesive sublimate residue appears to be a unique product of the freeze-drying of a dispersion, in water ice, of noncontacting particulates with a relatively high water solubility (Linke, 1965). A botryoidal, cohesive sublimate residue is formed when contacting particulates dispersed in water-ice droplets are sublimed in vacuo. When other materials such as rouge (ferric oxide) calcite, forsterite, graphite, and carbon black were used as particulate 
matter in otherwise duplicate experiments, no filamentary or botryoidal material was formed. Both of the filamentary sublimate residue substances that formed were siliceous and thus had a great affinity for water, relative to likely contaminants. Those substances which did not form filamentary or botryoidal sublimate residues were less hydrophilic (Meiter, 1963). In the case of the filamentary material, perhaps the subliming water vapor cleaned the impurities from the siliceous surfaces. This cleaning allowed the particle to be electrostatically attracted to and then bonded to the growing filament chain upon its release from the subliming ice (Fig. 2). This cleaning should take place as long as the solvent (water) wets the surface of the siliceous particle better than it does the contaminating molecules. The solubility product of amorphous silica in water is 0.003 at $0^{\circ} \mathrm{C}$ and 0.043 at $100^{\circ} \mathrm{C}$ (Linke, 1965). The solubility products of water with common contaminants that might be expected in the proposed environments (i.e., salts) are relatively high. The solubility product of silica with salts is relatively low. Thus, in most cases, the surface of the siliceous particle would have nothing but water on its surface while in the liquid dispersion.

For the "ice cleaning" to work, all the water has to do to clean the surface is to get between it and any contaminants-a separation distance of perhaps only a few molecular diameters (Fig. 4). The contaminating materials are dispersed or dissolved in the water and trapped there by rapid freezing until they are entrained by the water vapor wind as the ice sublimes. The concept of "ice cleaning" of the contacting surfaces may also be supported by the results of the experiment in which the clay was mixed "dry" with the ice particles in liquid nitrogen-no fluffy sublimate residue formed.

Ordinary dry montmorillonite particles (not otherwise cemented) cohere only loosely and will maintain an angle of repose of about $30^{\circ}$ on a centimeter scale. By con- trast, the sublimate residue from the 1000:1 montmorillonite dispersion supports vertical walls and even overhangs at the same scale. The bonds between particles are strong, flexible, and somewhat elastic. The flexible and even elastic behavior of the sublimate residue suggests that cementing between particles by deposits of salt on grain boundaries is unlikely. In the formation of "duricrust," water is "wicked" up to the soil surface by capillary action, where it evaporates, leaving the dissolved solids behind at the contact points between the individual soil particles. In the sublimate residues, salt bonding of the hexagonal clay particles would produce very fragile structures due to the large change in cross section of the bond relative to the cross section of the particle body. Only a bond strength that approached the strength of the clay particle itself could be strong enough to allow the large elastic deformations that were observed. We speculate that such strength can only be developed by silica-to-silica bonds. Also, migration of the salt to the joint between particles requires liquid water to be present on the surface at some point during the process. In the vacuum environment in which these experiments were performed the water existed in only two phases: as ice and as water vapor. Since most of the spheroids of ice in the liquid nitrogen were separate and were kept well below $-30^{\circ} \mathrm{C}$ and did not cohere prior to introduction into the vacuum chamber, any montmorillonite bonds which may have developed between the spheroids necessarily formed in the absence of liquid water.

It is possible, however, that during the experiment with the 100:1 montmorillonite solution, liquid water may have played a role in forming montmorillonite grain bonds within each individual "grape" of the aggregate prior to the droplets' freezing. The nature of the bonds thus formed is probably the same as the bonds between individual particles. In the case of the $100: 1$ botryoidal sublimate residue, the particles would have been "cleaned" by the action of the 
water before freezing. As the droplet was frozen the clay particles were in a sufficiently high concentration or the droplet was too big to freeze fast, and the clay particles found themselves touching one another in the ice matrix because of "zone" refining.

Zone refining refers to a process that occurs at an advancing ice crystal interface. The ice crystal prefers to include only water molecules in its crystalline matrix and therefore pushes any molecular and particulate impurities ahead of its growing interface. The impurities are trapped at the collisional interfaces between the ice crystals.

When sublimation from the $100: 1$ ice dispersion occurred, the monolayers of water that separated the clay particles were sublimed away, and the particles made molecular contact with one another before they could be released from the ice surface. This "clean" contact formed the same silica-tosilica bond that formed the filamentary sublimate residue. Thus, the only difference between the botryoidal sublimate residue and the filamentary sublimate residue is that the particles that form the filamentary residue have the opportunity to reorganize themselves into filaments, and the botryoidal particles remain in their relic structure.

Furthermore, surface water on the montmorillonite grains probably has little to do with maintaining the bonds between the relic spheres, although it conceivably could have played a role in the formation of the bonds. This is experimentally demonstrated by the fact that there is little or no change in the properties of the sublimate residue material after heating or upon exposure to vacuum, which should have removed the physisorbed monolayers of water.

The high electrical conductivity indicated by the SEM experiment suggests that static charges might be difficult to maintain within the sublimate residue and that electrostatic forces between particles might not be very important.

Perhaps Van der Waals forces or forces related to minimizing surface energy could be responsible for binding particles together. This seems unlikely given the high strength-to-weight ratio and the elastic properties.

We suggest that the contacting surfaces of the particles may be bonding directly. That is, the crystalline surfaces of the particles are "cold welding" or diffusion bonding together. In the botryoidal sublimate residue, it is likely that many of the clay particles in the initial 100:1 solution were touching each other (with only a few monolayers of water between them) prior to the freezing of the droplets, implying that the individual clay particles were arrayed in a "house-of-cards" structure (van Olphen, 1963). Thus, the sublimation of frozen water was preserving an already existing structure within each drop-an aerogel. Since the solid particles of clay were already touching in the presence of liquid water (that is, as the solutions were being frozen in the liquid nitrogen, and the particles were being pushed together as the droplet froze), the results of the sublimation of the $100: 1$ dispersion is necessarily somewhat different from that which presumably occurs in nature (i.e., Martian polar ices and comet nuclei) where the clay particles are believed not to be touching (Whipple, 1978, p. 67).

In the 1000: 1 mixtures of clay or Cabosil the particles apparently are not touching one another in the liquid or ice states. In the 100:1 mixtures the particles may not be touching in the liquid state but are most likely in contact within the ice droplet. As the droplet enters the liquid nitrogen, it freezes from the outside inward, into small radial crystals. As the ice crystals grow inward, they push impurities ahead of them (zone refining). The particles are trapped at the interference faces between the growing ice crystals. The soluble ions and gases are swept toward the center where they are trapped until the spherical ice droplet cracks from thermomechanical strain.

Unlike the 100:1 mixtures, evidence 
based on bulk densities and SEM images suggests that the sublimate residue produced by sublimation of the $1000: 1$ dispersion is reorganized - the structure and orientations assumed by the particles in the sublimate residue are different from those existing when the particles were in the ice. This reorganization strongly implies that most of the particles in the $1000: 1$ ice droplets were not in contact with one another prior to sublimation; instead, they were completely separated by ice, as the particles in Mars polar ice and comet ice probably are. Upon sublimation of the surrounding ice, the individual particles are unsupported so that some reconfiguration must occur. One possibility is that a newly freed particle might immediately encounter other such particulates within a few micrometers (Fig. 2). A spiderweb-like appearance of the aggregate produced with the $1000: 1$ water-clay mix is a probable indication that this process was dominant during the sublimation process, and that whatever bonds formed upon initial contact were sufficiently strong to prevent subsequent collapse. That these bonds are elastic, or at least flexible, is demonstrated by the formation of bubbles or blisters and the apparent occurrence of blowholes, all possibly formed by the exhausting water vapor, in the filamentary sublimate residue forming over the subliming ice (Straus and Schubert, 1977).

In the case of Martian polar and cometary sublimate residues, which form at temperatures well below freezing, one or more of the following three processes may be important:

(1) The natural materials may be clean from the start, in that contaminants may not be available at the time the water ice condenses onto the siliceous particle.

(2) The last monolayer of water on a surface remains mobile at temperatures well below $0^{\circ} \mathrm{C}$ [in fact two monolayers exhibit this property to temperatures far below those discussed here (Litvan, 1972)].

(3) Even at the temperatures at which no thermal mobility is possible (as with comet nuclei accreting far from the Sun), photon or other fluxes might induce significant mobility, given enough time.

Although there may be differences in the exact temperatures and pressures and dustto-ice ratios in nature, the bonds that formed between particles in the filamentary sublimate residue developed in essentially the same environment as would be expected at the polar caps of Mars or on the surface of a comet. Refractory particles previously separated by ice are brought together in the presence of water vapor at low pressures $(<0.2$ Torr) and temperatures $\left(<-25^{\circ} \mathrm{C}\right)$. Therefore, the properties of the botryoidal and filamentary sublimate residue (1000:1 and 100:1 initial mixtures) could be relevant to the sublimate residues of Martian polar cap and cometary surfaces.

In summary, we believe that the processes which might result in aggregated surface crusts on the Mars residual cap or on comets may be analogous to-but not identical to-those which occurred during our experiments. In our experiments, considerable opportunity exists for an attack of liquid $\mathrm{H}_{2} \mathrm{O}$ on the crystal surfaces. This opportunity may also exist in the case of the Mars residual cap during the hottest portion of the year, provided that the albedo is sufficiently low and salts are present. It should also be remembered that much longer times are available for such an attack to occur on Mars and on cometary surfaces. Furthermore, the high ion mobility which characterizes the first two monolayers of $\mathrm{H}_{2} \mathrm{O}$ at the ice-silicate interface (Litvan, 1972) may be sufficient, given enough time, to produce analogous effects. The maximum temperatures at which we believe these processes can operate on both the Mars residual cap and comets are $\sim 220$ to $230^{\circ} \mathrm{K}$, and mobility of the two silicate-ice monolayers persists to much lower temperatures. Finally, in the case of comets, the other forces involved in the evolution of the nucleus surface are so extremely weak (the 
gravity field is only $\sim 10^{-4} \mathrm{G}$, and the forces needed to eject grains are far less than what would be needed to eject our aggregates or to disrupt them) that processes at least analogous to those we observed in our experiments must be important, if only by default.

\section{ACKNOWLEDGMENTS}

The authors thank Eric G. Laue (of JPL), who continued the experiments up to the present; Dave D. Lawson (of JPL), who provided the solubility chemistry and IR spectroscopy data and analysis; Kenneth C. Evans (of JPL), who performed the exceptional scanning electron microscopy; Cindy Inouye (of JPL), who performed the $\mathrm{X}$-ray diffraction analysis; and Mike Gaffey (of Rensselear Polytechnic Institute), who performed an in-depth review of this paper. This research was conducted at the Jet Propulsion Laboratory, California Institute of Technology, under contract with the National Aeronautics and Space Administration.

\section{REFERENCES}

BrownleE, D. E. (1978). Microparticle studies by sampling techniques. In Cosmic Dust (J. A. McDonnell, Ed.), p. 317. Wiley, New York.

Cutts, J. A., K. R. Blasius, and W. J. Roberts (1979). Evolution of Martian polar landscapes: In- terplay of long-term variations in perennial ice cover and dust storm intensity. J. Geophys. Res. 84(B6), 2975-2994.

Fanale, F. P., and J. R. Salvail (1984). An idealized short-period comet model: Surface insolation, $\mathrm{H}_{2} \mathrm{O}$ flux, dust flux, and mantle evolution. Icarus 60 , 476-511.

LINKE, W. F. (1965). Solubilities of Inorganic and Metal-Organic Compounds, $I$, 4th ed. Amer. Chem. Soc., Washington, D.C.

Litvan, G. G. (1972). Phase transitions of adsorbates. III. Heat effects and dimensional changes in nonequilibrium temperature cycles. $J$. Colloid Interface Sci. 38(1), 75-83.

Meiter, L. (1963). Handbook of Analytical Chemistry, 1st ed. McGraw-Hill, New York.

Scheidegger, A. E. (1974). The Physics of Flow Through Porous Media, 3rd ed., pp. 243-245. Univ. of Toronto Press, Toronto.

Straus, J. M., and G. Schubert (1977). Thermal convection of water in a porous medium: Effects of temperature- and pressure-dependent thermodynamic and transport properties. J. Geophys. Res. 82.

van Olphen, H. (1963). An Introduction to Clay Colloid Chemistry, p. 301. Interscience, New York.

Weaver, C. E., And L. D. Pollard (1973). The Chemistry of Clay Minerals (Developments in Sedimentology No. 15), p. 213. Elsevier, New York.

Whipple, F. L. (1978). Comets. In Cosmic Dust (J. A. McDonnell, Ed.). Wiley, New York. 